\title{
A Parameterisation of $\gamma$-ray production cross-sections for pp interactions in a broad energy range
}

\author{
Andrew M. Taylor ${ }^{\mathrm{a}}$ \\ Dublin Institute for Advanced Studies, 31 Fitzwilliam Place, Dublin 2, Ireland
}

\begin{abstract}
Using different Monte Carlo codes such as Geant 4.10, Pythia 8.18, SIBYLL and QGSJET, as well as compiling published data on $p p$ interactions close to the kinematic threshold, we parametrize the energy spectra and production rates of gamma-rays by simple but quite accurate $(\leq 20 \%)$ analytical expressions in a broad range from the kinematic threshold to $\mathrm{PeV}$ energies.
\end{abstract}

\section{Introduction}

High energy astrophysics bridges several major disciplines in physics, broadly drawing on solid foundations in particle physics enabling new areas in high energy astrophysics to be investigated. The nature of the detection methods which high energy $\gamma$-ray telescopes utilise, dictated by the particle physics of high energy interactions, demand a firm understanding of the different processes involved. Limitations in our understanding of particle physics interactions, such as the $p p \rightarrow A B+\pi^{0} \gamma$-ray production channel, therefore limit the depth with which the astrophysical environments may be probed.

Presently, the restricted energy range for which the pion production cross-section and the differential crosssection for the $\gamma$-ray secondaries have been probed forces the use of descriptions beyond the energy range for which they have been tested ranges. This fact, coupled to the large proton energy range which astrophysical processes probe via this process, and the multitude of Monte Carlo extrapolations available, motivate a simple analytic template capable of encapsulating these descriptions. A more detailed account of the $\gamma$-ray production description found is provided in [1].

\section{2. p-p interaction descriptions}

Presently, a host of phenomenological approaches exist with which one may calculate hadronic interactions in both perturbative and non-perturbative regimes. Sophisticated Monte Carlo (MC) codes have been developed which handle complex calculations and predict many quantities that agree well with experimental data. Accompanying these numerical descriptions, ref. [2] used the results of publicly available codes to find an accurate parametrization of different secondary particle spectra at high proton energies, with $0.1 \leq T_{p} \leq$ $10^{5} \mathrm{TeV}$. With the $<10 \mathrm{GeV}$ component of astrophysical $\gamma$-ray sources becoming probeable by present and

a e-mail: taylora@cp.dias.ie next-generation instruments, the limitations in energy range of this parametrization are becoming apparent. Therefore, a parametrization that is both accurate and that spans from threshold up to very high energies is needed. Moreover, very hot astrophysical plasma calculations as well as the increasing sensitivity of the $\gamma$-ray instruments such as Fermi-LAT satellite, which has recently observed $\gamma$-ray spectra that reveal a sub-GeV bump, require accurate $\gamma$-ray production cross sections at low energies near the $\mathrm{p}-\mathrm{p}$ kinematic threshold.

\section{Inclusive $\pi^{0}$ production cross section and multiplicity}

The theoretically motivated parametrization of the high energy $p-p$ inelastic cross section has a quadratic functional form of the logarithm of the proton energy. We take here the total and elastic p-p cross section data compiled by the Particle Data Group (PDG) [3]. These data include the recent measurements at center-of-mass energies $\sqrt{s}=7$ and $8 \mathrm{TeV}$ published by the TOTEM collaboration at the LHC [see e.g. 4,5]. We suggest the following parametrization for the $\mathrm{p}-\mathrm{p}$ total inelastic cross section

$$
\begin{aligned}
\sigma_{\text {inel }}= & {\left[30.7-0.96 \log \left(\frac{T_{p}}{T_{p}^{\mathrm{th}}}\right)+0.18 \log ^{2}\left(\frac{T_{p}}{T_{p}^{\mathrm{th}}}\right)\right] } \\
& \times\left[1-\left(\frac{T_{p}^{\mathrm{th}}}{T_{p}}\right)^{1.9}\right]^{3} \mathrm{mb} .
\end{aligned}
$$

Here, $T_{p}$ is the proton kinetic energy in the laboratory frame, $T_{p}^{\mathrm{th}}=2 m_{\pi}+m_{\pi}^{2} / 2 m_{p} \approx 0.2797 \mathrm{GeV}$ is the threshold kinetic energy. $m_{p}$ and $m_{\pi}$ are the proton and $\pi^{0}$ masses, respectively. The units that we use throughout are the natural units (i.e. $\hbar=c=k_{B}=1$ ).

Figure 1 compares the parametrization of the Fig. 1 against two other parametrizations [2] and [6] often used in astrophysical contexts, as well as the observational data from [3].

This is an Open Access article distributed under the terms of the Creative Commons Attribution License 4.0, which permits unrestricted use, distribution, and reproduction in any medium, provided the original work is properly cited. 


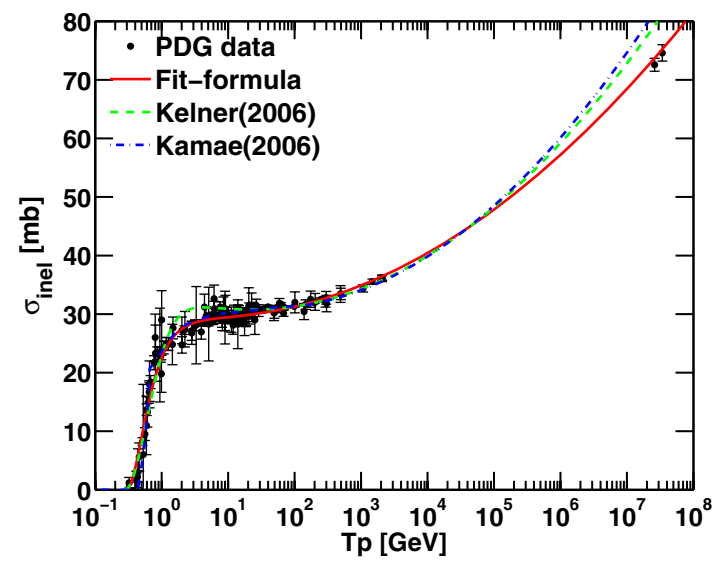

Figure 1. The total $p-p$ inelastic cross section as a function of the proton kinetic energy in the laboratory system. The data points are taken from the PDG [3] cross section data. The red line is our $\chi^{2}$-fit formula shown in Fig. 1. The dashed green line is the formula given in [2] and the dot-dashed blue line is the formula given in [6].

To obtain the inclusive $\pi^{0}$ production cross section or average multiplicity, we have used both experimental data and calculations from MC codes, which are themselves tuned to describe experimental data over a given energy range.

For proton kinetic energies $T_{p} \leq 2 \mathrm{GeV}, \pi^{0}$ production is dominated by baryon resonance production. The leading $\pi^{0}$ production channels for these energies are $p p \rightarrow$ $p p \pi^{0}, \quad p p \rightarrow p p 2 \pi^{0}$ and $p p \rightarrow\{p n, D\} \pi^{+} \pi^{0}$. Other two-pion and three-pion channels are negligible. Good quality data exist for these channels in this energy region, which we have here compiled.

For energies $T_{p}>2 \mathrm{GeV}$, we calculate the average $\pi^{0}$ production multiplicity using Geant 4.10.0, Pythia 8.18, SIBYLL 2.1 and QGSJET-I. Note that the latest version of the QGSJET description presently available is QGSJET-II. We have not run either SIBYLL or QGSJET codes here, we instead rely on the fits of $\pi^{0}$ and $\eta$ mesons spectra for both SIBYLL 2.1 and QGSJET-I provided in [2], valid for $T_{p}>100 \mathrm{GeV}$.

The numerical descriptions we run are those provided in Geant 4.10.0 and Pythia 8.18. Geant 4.10.0 is itself actually a toolkit for the simulation of the passage of particles through matter. Thus it does not stand in any of the MC code classes. However, Geant 4.10.0 contains sophisticated hadronic interaction models such as "FTFP_BERT", that we use here, which implements Bertini-style cascade at low energies $T_{p} \leq 5 \mathrm{GeV}$. For $4<T_{p} \leq 10^{5} \mathrm{GeV}$ it includes the FRITIOF string model, see $[7,8]$. Simulations with Pythia 8.18 are done by using the default Pythia 8.18 tune and selecting "SoftQCD" processes.

In this way, we adopt the Geant 4.10 .0 average $\pi^{0}$ production multiplicity to fill in the energy gap between the experimental data $T_{p} \leq 2 \mathrm{GeV}$ and the high energy models adopted. At high energies, different hadronic models predictions start to diverge. Thus, at these high energies we provide the option to adopt the multiplicity from one of these hadronic models: namely Geant 4.10.0; Pythia 8.18; SIBYLL 2.1 and QGSJET-I.
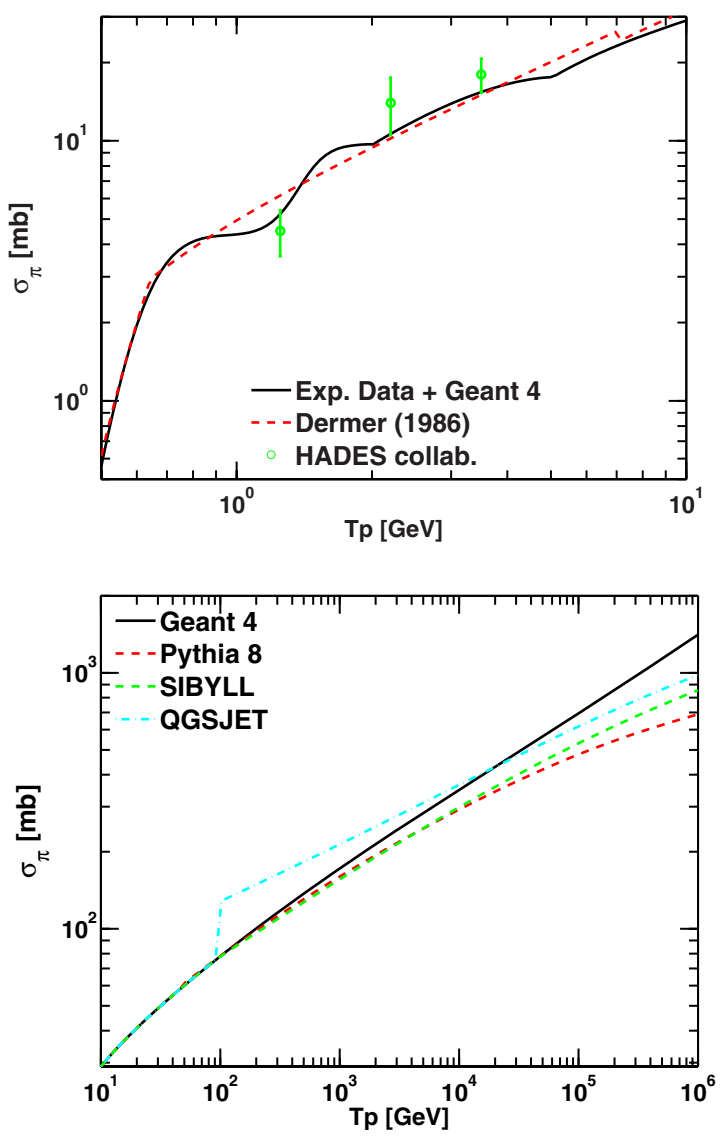

Figure 2. Inclusive $\pi^{0}$ production cross section as a function of proton kinetic energy. The left panel compares the parametrization given in Ref. [9], our parametrization and the experimental data at $1.25,2.2$ and $3.5 \mathrm{GeV}$ from the HADES collaboration [10-12]. The panel on the right shows the differences between high energy models using the parametrization we have introduced here. Notice that Geant 4.10.0 works for $T_{p} \leq 10^{5} \mathrm{GeV}$, in the plot we have extrapolated its multiplicity up to $T_{p}=10^{6} \mathrm{GeV}$.

We have fitted the inclusive $\pi^{0}$ production cross sections and multiplicities from kinematic threshold to very high energies $\left(T_{p} \sim 1 \mathrm{PeV}\right)$ and parametrized their descriptions. Figure 2 compares our low energy $\sigma_{\pi}$ values with the parametrization provided in Ref. [9] and the data from the HADES collaboration [10-12].

\section{Parametrization of the $\gamma$-ray differential cross section}

We divide the $\gamma$-ray differential cross section into two parts. The fist part is the maximum value $A_{\max }\left(T_{p}\right)=$ $\max \left(d \sigma / d E_{\gamma}\right)$ that depends only on the proton energy $T_{p}$. The second part is $F\left(T_{p}, E_{\gamma}\right)$ which describes the shape of the spectrum and is a function of $T_{p}$ and $\gamma$-ray energy $E_{\gamma}$. We therefore express the differential cross section as,

$$
\frac{d \sigma}{d E_{\gamma}}\left(T_{p}, E_{\gamma}\right)=A_{\max }\left(T_{p}\right) \times F\left(T_{p}, E_{\gamma}\right) .
$$

The peak $A_{\max }\left(T_{p}\right)$ is a function of the pion production cross section and is fitted separately from $F\left(T_{p}, E_{\gamma}\right)$. Let 
us define the following variables:

$$
\begin{aligned}
& Y_{\gamma}=E_{\gamma}+\frac{m_{\pi}^{2}}{4 E_{\gamma}}, \quad Y_{\gamma}^{\max }=E_{\gamma}^{\max }+\frac{m_{\pi}^{2}}{4 E_{\gamma}^{\max }}, \\
& X_{\gamma}=\frac{Y_{\gamma}-m_{\pi}}{Y_{\gamma}^{\max }-m_{\pi}} .
\end{aligned}
$$

Here, $E_{\gamma}^{\max }$ is the maximum $\gamma$-ray energy allowed by the kinematics. Let us denote with $E_{\pi}^{C M}$ and $E_{\pi L A B}^{\max }$ the maximum $\pi^{0}$ total energy in the center-of-mass and laboratory systems, respectively. Let us further denote with $\gamma_{C M}$ the Lorentz factor of the center-of-mass system and $\gamma_{\pi}^{L A B}$ the maximum $\pi^{0}$ Lorentz factor in the laboratory system. The maximum or minimum $\gamma$-ray energy $E_{\gamma}^{\max / \min }$ is given by:

$$
\begin{aligned}
E_{\pi}^{C M} & =\frac{s-4 m_{p}^{2}+m_{\pi}^{2}}{2 \sqrt{s}}, \\
E_{\pi L A B}^{\max } & =\gamma_{C M}\left(E_{\pi}^{C M}+P_{\pi}^{C M} \beta_{C M}\right), \\
\gamma_{C M} & =\frac{T_{p}+2 m_{p}}{\sqrt{s}}, \gamma_{\pi}^{L A B}=\frac{E_{\pi L A B}^{\max }}{m_{\pi}}, \\
E_{\gamma}^{\max / \min } & =\frac{m_{\pi}}{2} \gamma_{\pi}^{L A B}\left(1 \pm \beta_{\pi}^{L A B}\right) .
\end{aligned}
$$

Here, $s=2 m_{p}\left(T_{p}+2 m_{p}\right), P_{\pi}^{C M}=\sqrt{\left(E_{\pi}^{C M}\right)^{2}-m_{\pi}^{2}}$ is the pion center-of-mass momentum, $\beta_{C M}=\sqrt{1-\gamma_{C M}^{-2}}$ is the center-of-mass velocity, and $\beta_{\pi}^{L A B}=\sqrt{1-\left(\gamma_{\pi}^{L A B}\right)^{-2}}$ is the pion maximum velocity in the laboratory system.

The value of $Y_{\gamma}^{\max }$ is the same if we replace the maximum $\gamma$-ray energy with the minimum one $E_{\gamma}^{\min }$. The reason being that $Y_{\gamma}$ is a symmetric function of $E_{\gamma}$ with respect to $E_{\gamma}=m_{\pi} / 2$ on a logarithmic energy scale. Values of $Y_{\gamma}$ lie in $Y_{\gamma} \in\left[m_{\pi}, Y_{\gamma}^{\max }\right]$, thus, $X_{\gamma} \in$ $[0,1]$. It is expected that $F\left(T_{p}, E_{\gamma}\right)$ be a function of $Y_{\gamma}$, hence, a function of $X_{\gamma}$. Therefore, the functional shape of $F\left(T_{p}, X_{\gamma}\right)$, for a given proton energy, is defined in the range $[0,1] \times[0,1]$, which simplifies the expression of its parametrization.

With very good accuracy $F\left(T_{p}, E_{\gamma}\right)$ can be parametrized as

$$
F\left(T_{p}, E_{\gamma}\right)=\frac{\left(1-X_{\gamma}^{\alpha\left(T_{p}\right)}\right)^{\beta\left(T_{p}\right)}}{\left(1+\frac{X_{\gamma}}{C}\right)^{\gamma\left(T_{p}\right)}} .
$$

Expressions for $\alpha\left(T_{p}\right), \beta\left(T_{p}\right), \gamma\left(T_{p}\right)$, and $C$ are provided in [1].

\subsection{Gamma-ray spectra from Geant 4.10.0, Pythia 8.18, SIBYLL 2.1 and QGSJET-I}

Using the parametrized results obtained, the determination of the resultant $\gamma$-ray spectrum produced through $\mathrm{p}-\mathrm{p}$ interactions with a power-law distribution of protons is considered. To account for the energy range of the protons,

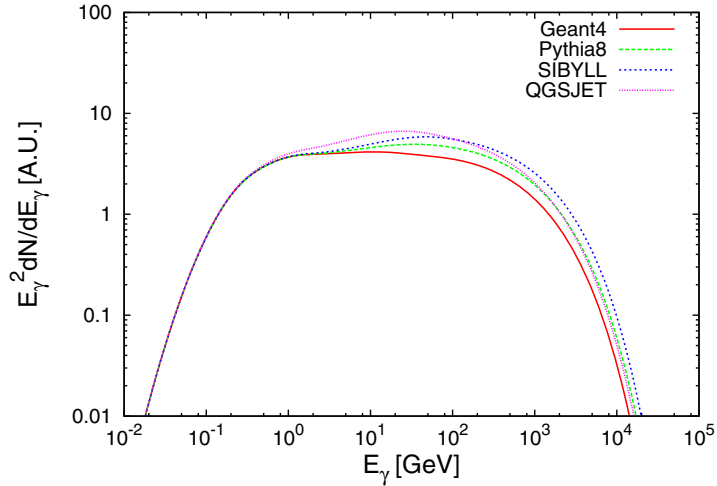

Figure 3. The resulting $\gamma$-ray energy flux from a power-law proton cosmic-ray flux with a maximum energy of $p_{\max }=$ $10^{14} \mathrm{eV}$, a spectral slope of $\alpha=2$, and cutoff strength $\beta=1$, for the different hadronic models considered.

and their abundance at each energy, an integral over the proton energy spectra must be carried out,

$$
\Phi_{\gamma}\left(E_{\gamma}\right)=4 \pi n_{\mathrm{H}} \int \frac{d \sigma}{d E_{\gamma}}\left(T_{p}, E_{\gamma}\right) J\left(T_{p}\right) d T_{p},
$$

where $n_{\mathrm{H}}$ is the density of target protons.

For a proton spectrum of the form

$$
J_{p}\left(p_{p}\right)=\frac{A}{p_{p}^{\alpha}} \exp \left[-\left(\frac{p_{p}}{p_{p}^{\max }}\right)^{\beta}\right]
$$

the corresponding $\gamma$-ray spectrum produced may therefore be obtained. Figure 3 is provided as an example case, demonstrating the range of spectra that may be produced between the different descriptions considered for the specific case of $p_{\max }=10^{14} \mathrm{eV}$, a spectral slope of $\alpha=2$, and cutoff strength $\beta=1$.

\section{Discussion and conclusions}

We provide an accurate and simple fitting formula framework for the $\gamma$-ray spectra produced in $\mathrm{p}-\mathrm{p}$ inelastic collisions that cover the energy interval from threshold to very high energies $\left(T_{p} \sim 1 \mathrm{PeV}\right)$, with the added flexibility to switch between different high energy models. As space-based $\gamma$-ray detectors are beginning to probe the $E_{\gamma}<100 \mathrm{MeV}$, improved accuracy of the $p p \rightarrow \gamma$ production cross sections at low energies are needed. As a result we have revised here the low energy $\pi^{0}$ production cross section and differential cross section data below $2 \mathrm{GeV}$. We use Geant 4.10 .0 for energies above $2 \mathrm{GeV}$ and at high energies, where different hadronic interaction model descriptions diverge, we have used Geant 4.10.0, Pythia 8.18, SIBYLL 2.1 and QGSJETI. Different validations of the MC codes against the $\pi^{0}$ and $\gamma$-ray experimental data at the LHC energies (see e.g. [13-16]) show that none of the MC models is able to explain fully all the available experimental data. It is for this reason that we parametrize different models which are representative of different descriptions of hadronic interactions. Note that the latest version of QGSJET in the CORSIKA code is QGSJET-II. 
Recently the TOTEM collaboration at the LHC have measured the $\mathrm{p}-\mathrm{p}$ inelastic cross section at $\sqrt{s}=7$ and $8 \mathrm{TeV}$. The widely used parametrizations for this cross section in astrophysics e.g. [2,6], do not fit well these two data points. Therefore, here we give a parametrization of the $\mathrm{p}-\mathrm{p}$ inelastic cross section that fits well both the low energy as well as new high energy LHC data.

The inclusive $\pi^{0}$ production cross section that we have suggested here, is composed of fits of the experimental one-pion and two-pion production data and fits from Geant 4.10.0 $\pi^{0}$ production multiplicity. At very high energies we have fitted separately the $\pi^{0}$ production multiplicity from Geant 4.10.0, Pythia 8.18, SIBYLL 2.1 and QGSJET-I. All these fits have an accuracy better than $3 \%$ for proton energy $T_{p}^{\text {th }} \leq T_{p} \leq 1 \mathrm{PeV}$. It is very likely that this parametrization continues to accurately describe the inclusive $\pi^{0}$ production cross section above $1 \mathrm{PeV}$.

\section{References}

[1] E. Kafexhiu, F. Aharonian, A.M. Taylor, G.S. Vila, Physical Review D 90, 123014 (2014), 1406.7369

[2] S.R. Kelner, F.A. Aharonian, V.V. Bugayov, Physical Review D 74, 034018, (2006), arXiv: astro-ph/0606058

[3] J. Beringer, J.-F. Arguin, R. M. Barnett, K. Copic, O. Dahl, D.E. Groom, C.-J. Lin, J. Lys, H. Murayama, C. G. Wohl et al., Physical Review D 86, 010001 (2012)

[4] G. Antchev et al. (TOTEM), Europhys. Lett. 101, 21004 (2013)

[5] G. Antchev et al. (TOTEM Collaborations), Phys. Rev. Lett. 111, 012001 (2013)

[6] T. Kamae, N. Karlsson, T. Mizuno, T. Abe, T. Koi, Astrophysical Journal 647, 692 (2006)
[7] S. Agostinelli, J. Allison, K. Amako, J. Apostolakis, H. Araujo, P. Arce, M. Asai, D. Axen, S. Banerjee, G. Barrand, et al., Nuclear Instruments and Methods in Physics Research A 506, 250 (2003)

[8] J. Allison, K. Amako, J. Apostolakis, H. Araujo, P. Arce Dubois, M. Asai, G. Barrand, R. Capra , S. Chauvie, R. Chytracek, et al., IEEE Transactions on Nuclear Science 53, 270 (2006)

[9] C.D. Dermer, Astrophysical Journal 307, 47 (1986)

[10] G. Agakichiev, A. Balanda, D. Belver, A.V. Belyaev, A. Blanco, M. Böhmer, J.L. Boyard, P. BraunMunzinger, P. Cabanelas, E. Castro et al., Physics Letters B, 690, 118 (2010), 0910.5875

[11] G. Agakishiev, A. Balanda, D. Belver, A. Belyaev, A. Blanco, M. Böhmer, J.L. Boyard, P. Cabanelas, E. Castro, J.C. Chen et al., European Physical Journal A 48, 64 (2012), 1112.3607

[12] G. Agakishiev, H. Alvarez-Pol, A. Balanda, et. al., European Physical Journal A 48, 74 (2012), 1203.1333

[13] D. d'Enterria, R. Engel, T. Pierog, S. Ostapchenko, K. Werner, Astroparticle Physics 35, 98 (2011), 1101.5596

[14] O. Adriani, L. Bonechi, M. Bongi, G. Castellini, R. D’Alessandro, K. Fukatsu, M. Haguenauer, T. Iso, Y. Itow, K. Kasahara, et al., Physical Review D 86, 092001 (2012), 1205.4578

[15] O. Adriani, L. Bonechi, M. Bongi, G. Castellini, R. D’Alessandro, K. Fukatsu, M. Haguenauer, T. Iso, Y. Itow, K. Kasahara, et al., Physics Letters B 715, 298 (2012), 1207.7183

[16] H. Menjo, O. Adriani, M. Bongi, G. Castellini, R. D’Alessandro, K. Fukatsu, M. Haguenauer, Y. Itow, K. Kasahara, K. Kawade, et al., Nuclear Instruments and Methods in Physics Research A 692, 224 (2012) 